\title{
1 Common Themes in Centriole and Centrosome Movements
}

2

3 Sue Vaughan ${ }^{1}$ and Helen R. Dawe ${ }^{2}$

4

5

$6{ }^{1}$ School of Life Sciences, Oxford Brookes University, Gipsy Lane, Oxford, OX3 0BP, UK

$7 \quad{ }^{2}$ Biosciences, College of Life and Environmental Sciences, University of Exeter, Stocker 8 Road, Exeter, EX4 4QD, UK

9

10 Corresponding author: Dawe, H. R. (h.r.dawe@exeter.ac.uk) 


\section{Abstract}

12 Centrioles are found in nearly all eukaryotic cells and are required for growth and

13 maintenance of the radial array of microtubules, the mitotic spindle, and cilia and flagella.

14 Different types of microtubule structures are often required at different places in a given cell;

15 centrioles must move around to nucleate these varied structures. Here we draw together

16 recent data on diverse centriole movements to decipher common themes in how centrioles

17 move. Par proteins establish and maintain the required cellular asymmetry. The actin

18 cytoskeleton facilitates movement of multiple basal bodies. Microtubule forces acting on the

19 cell cortex, and nuclear-cytoskeletal links, are important for positioning individual

20 centrosomes, and during cell division. Knowledge of these common mechanisms can inform

21 the study of centriole movements across biology.

\section{Introduction}

In metazoan cells the major microtubule organising centre (MTOC) of the cell is the centrosome (Figure 1 and glossary). It is composed of a pair of microtubule-based centrioles surrounded by a pericentriolar matrix (PCM). Centrioles were once thought to be static organelles located in the centre of the cell, hence their name. In fact, they move around the cell to fulfil their functions and correct centriole and centrosome positioning is vital for many biological processes. There are now many examples of centriole/centrosome movements in 30 various physiological contexts and many different cell types. We will use the term 31 centrosome in situations where the centriole pair and PCM all move together, and centriole where only a single centriole moves. Basal body is the term often used for a centriole that assembles a cilium or flagellum. While many advances have been made over the last few

34 years in understanding what controls centrosome, centriole and basal body position, often, 35 these fields are investigated in isolation. If the data from these disparate fields are analysed 
together, it becomes apparent that there are common themes in both the mechanics of movement and the regulatory mechanisms involved. Here, we first provide a brief overview of the contexts in which centriole, centrosome and basal body movements are seen, and then elaborate on how some of the common themes that are emerging across eukaryotic biology are applied in each case.

Centrosome location is critical for many biological processes (Figure 2-4) and also impacts the position of other organelles. The centrosome and the nucleus are closely associated, and the Golgi apparatus is also found near the centrosome [1] enabling polarization of membrane trafficking and secretory machineries [2-3]. While centrosomes are not absolutely required to organise the mitotic spindle [4-6], their position is important in symmetric and asymmetric cell divisions (Figure 3) as movement of the two centrosomes to opposite sides of the nucleus defines both the axis of division, and spindle position. In Caenorhabditis elegans, centrosome positioning is key to the polarity establishment required during asymmetric cell divisions and defines the anterior-posterior axis of the embryo [7]. The African trypanosome does not use its centrioles (located at the base of the flagellum and termed basal bodies) to organise the mitotic spindle; however, basal body positioning and segregation control cell morphogenesis by influencing cytoskeletal construction and directly positioning the kinetoplast

54 (mitochondrial genome) [8] (Figure 3).

Centriole/centrosome position also contributes to the spatial organisation of many cells in

57 G1/interphase. In the biflagellate green alga Chlamydomonas reinhardtii centriole/centrosome position maintains overall cell geometry [9], and in metazoans it organises the radial microtubule array during interphase (Figure 2). In many types of 60 migrating cells, centrosome position between the nucleus and the leading edge is key to 
61 migration [10-13]. Formation of the specialised immunological and virological synapses involves centrosome re-orientation. During immunological synapse formation, the centrosome migrates to the contact site between the T-cell and the antigen presenting cell (Figure 4), where, in cytotoxic T-cells, it is involved in directed secretion of lytic granules [14]. During virological synapse formation, which mediates the cell-cell transfer of viral particles between an infected cell and a target cell, the centrosome of the infected cell likewise re-orients towards the site of contact between the cells [15]. Finally, while centrioles are not absolutely essential for cell division [16], they are critical for ciliogenesis. Humans build motile and sensory cilia, and ciliogenesis of both kinds requires centriole/basal body movement to the cell surface [17] (Figures 2 and 4). Most branches of the tree of life are ciliate, and in many cases failure to build a cilium is incompatible with life. Even in Drosophila, which can develop without centrioles, death eventually occurs due to the lack of cilia on sensory neurons [16].

Thus, there is a considerable diversity of centriole/centrosome movements in biology. All of the studied mechanisms of centriole movement require the actin or microtubule cytoskeletons, or both. Are there common mechanisms that apply across these varied processes or are there specialised mechanisms to facilitate movement in each physiological context?

\section{The microtubule cytoskeleton and centriole movement}

82 The position of the centrosome in both migrating and non-migrating interphase cells requires a polarised radial microtubule array [18-19]. During cell migration, centrosome position is actin-independent $[10,12]$, while the cortical pool of the microtubule minus-end directed motor cytoplasmic dynein is implicated in centrosome position in several different cell types 
$86[10,12-13,20]$. During astrocyte migration, the small GTPase Cdc42 controls centrosome

87 and Golgi re-orientation towards the direction of migration through the microtubule cytoskeleton and cytoplasmic dynein $[10,21]$; however, in fibroblasts, the centrosome remains at the cell centre while the nucleus moves rearward [20]. Nonetheless, centrosome maintenance at this position is dependent on microtubules and dynein as inhibition of either

91 causes a rearward centrosome displacement [20].

Microtubules and cytoplasmic dynein are also implicated in centrosome positioning in nonmigrating cells $[19,22-24]$, suggesting that, when the centrosome needs to be central within the cell, its position is actively maintained using microtubules and dynein to stabilise the centrosome-associated microtubule array. In interphase cells, the centriole pair do not necessarily remain together. The older, mature, centriole can remain stationary while the younger, immature centriole moves around the cell [25]. The Rho-associated kinase p160Rock, is proposed to regulate the central position of the mature centriole [26]. p160Rock is a major regulator of myosin II but it has many different substrates [27] and during cell migration it can mediate both microtubule-dependent centrosome re-orientation [28] and actin rearrangements; consequently, the way in which it affects centrosome position remains unclear.

In summary, microtubules and cytoplasmic dynein regulate centrosome position when a single centrosome needs to be positioned in the centre of the cell. How about when centrioles need to move away from the centre, or if there are multiple centrioles to move? In these cases, several studies have highlighted a primary role for the actin cytoskeleton in centriole positioning. 


\section{Actin in centrosome/centriole movements}

112 While the first cell division of many organisms is symmetric, that of C. elegans is

113 asymmetric. During the first division of the C. elegans zygote, the cell divides

114 asymmetrically along its anterior-posterior axis to give rise to two cells that are committed to

115 different cell fates. The centriole pair are key factors in the polarity establishment required

116 during these divisions. They are derived from the sperm cell rather than the oocyte and

117 become embedded in the actin cortex underlying the plasma membrane by a mechanism that

118 may not involve interactions with microtubules. RNAi ablation of tubulin does not prevent

119 polarity establishment and is not required for centriole-cortex interaction [7, 29], suggesting

120 that another cytoskeletal polymer mediates centriole position. However, another study found

121 that tubulin disruption delays polarity induction, which requires a small centrosomal

122 microtubule aster [30]; therefore the precise role of microtubules in this process remains to be

123 clarified.

124

125 The trachea, oviduct and ependymal epithelium of mammals are composed of a multi-ciliated

126 epithelium where each cell may have hundreds of cilia, each grown from a basal body. These

127 form de novo and migrate simultaneously to the apical cell surface. Drugs that target

128 microtubules do not directly stop basal body movement in oviduct [31], although the

129 contribution of microtubules to basal body movements in other cell types is unclear. In

130 contrast, much evidence from several cell types implicates the actin cytoskeleton in basal

131 body migration and docking at the cell surface. Actin and myosin associate with either basal

132 bodies, or the material surrounding them [32-34] and basal body migration is blocked by

133 treatment with inhibitors of actin [35] or myosin [36]. Basal body docking with the cell

134 membrane is also actin dependent. The Wnt planar cell polarity pathway and its effectors are

135 implicated in membrane trafficking during ciliogenesis and the formation of an actin array 
essential for basal body docking [37-39] [40]. Finally, myosin II localises to basal body accessory structures in multi-ciliated epithelia [32], and is needed for basal body migration [41].

140 There are emerging similarities in the structure and function of basal bodies of cilia and 141 centrioles at the immunological synapse [42], such as the requirement for intraflagellar transport components. These were previously thought of as cilium assembly and maintenance proteins [43], but recent data have also demonstrated a role in polarised recycling at the

144 immunological synapse [44]. This suggests that there may be similar principles guiding 145 centriole movement and membrane trafficking in both cases. During immunological synapse

146 formation, receptor-mediated engagement between the immune cell and its target triggers a 147 transient aggregation of actin across the nascent synapse [45]. The polarisation of the

148 centriole pair to the synapse is accompanied by concomitant actin clearance from the inner 149 part of the forming synapse, to produce an outer ring of actin $[14,46]$ and it has been 150 suggested that the forces generated by actin clearance are used to move the centrioles forward

151 [2].Centrioles are always docked at the centre of the synapse and it is unclear whether this is due to radial actin reorganisation that localises them to this region by default, or whether there are other actin binding proteins that regulate the site of docking. Much less is known

154 about the role of the cytoskeleton in virological synapse formation, although it is an actin155 dependent process [47] and integrity of both the actin and microtubule cytoskeletons is 156 crucial [48-49]. In general it is over-simplistic to consider the actin and microtubule cytoskeletons in isolation. Extensive cross-talk exists and many biological processes are carried out by actin 
and microtubules acting together. Much research has shown that interactions of microtubules that are anchored into the actin cortex are often responsible for centriole positioning.

\section{Microtubule interactions with the actin cortex and centriole and centrosome positioning}

164 Interaction between microtubules and the actin cytoskeleton at the cell cortex are essential

165 for maintaining the physical position of the centrosome within the cell and for orchestrating 166 placement of the duplicated centrosomes during symmetric and asymmetric cell divisions.

167 Pulling forces of microtubules anchored to the cell cortex provide a mechanism for 168 centrosome positioning $[11,50]$. Studies of male germline stem cells in Drosophila have 169 revealed differential centrosome behaviour during the asymmetric cell divisions that 170 characterise development. After centrosome duplication, the older of the two centrosomes 171 retains a well-defined microtubule array and remains in place, while the younger centrosome 172 nucleates few microtubules and migrates away to set up the symmetrical plane of the mitotic 173 spindle [51].

175 The pulling forces are provided by dynein on microtubules that extend to and are anchored into the cell cortex regulated by both dynein and the actin motor myosin II. Myosin II is one of several proteins that organise the cortex, and cortical organisation is critical for providing the mechanical support needed for centrosome positioning. The importance of cortical

179 rigidity has been highlighted by recent studies that showed that myosin II- and moesin180 dependent cortical rigidity are required for spindle positioning [52-54]. Moreover, cell shape 181 plays an important part in spindle orientation, which is highly dependent on cell-substrate adhesions [55] that are communicated to the cytoskeleton via integrins and actin-microtubule linkers including EB1 and myosin X [56-57]. 
185 Taken together, these studies provide evidence for a conserved pathway that explains centrosome movement in cells containing a centralised centrosome with a radial array of

187 microtubules that are in contact with the plasma membrane. However, not all eukaryotic cells 188 have a radial array of microtubules emanating from a centralised MTOC. Protists such as the

189 African trypanosome represent the polar opposite, with microtubules arranged as a 190 subpellicular sheet underlying the plasma membrane [58]. Basal bodies extend a microtubule axoneme for the flagellum in this organism, but not a radial array of microtubules within the cell body. Despite these differences, microtubules do provide the mechanism for basal body movements and segregation during cell division [59] and these microtubules are closely associated with the plasma membrane in the same way that microtubules associate with the actin cortex underlying the plasma membrane in other organisms.

197 Thus, the idea that microtubule interactions with a cortical cytoskeleton are used to move single or paired centrioles/centrosomes is conserved across eukaryotic biology.

\section{The role of the nuclear envelope in centriole movements.}

201 It is increasingly recognised that the nucleus, as well as the cortex, plays a role in positioning centrioles. In many cells, the centrioles are tightly associated with the nuclear envelope. This connection is observed in lower eukaryotes as a physical linkage of striated fibres called the

204 basal body-nucleus connector (rhizoplast, [60]). During the cell division cycle of

205 Chlamydomonas, the two flagella are cleaved from the cell. The two pairs of centrioles move 206 from the apical surface to the poles of the spindle during mitosis, and after division return to 207 the apical surface and grow two new flagella. The basal body-nucleus connector exists as 208 striated fibres that connect both pairs of centrioles, which are in turn connected to the nucleus 209 by centrin-containing rhizoplasts [61]. Is the nucleus, the centriole or both involved in the 
210 movement to and from the apical surface? In cells lacking the rhizoplast connection, the

211 nucleus is mispositioned, but the mature centriole is correctly localised, indicating that

212 nuclear mis-positioning has little impact on mature centriole positioning [9] and suggesting

213 that the centriole regulates nuclear positioning rather than vice versa. In a migrating

214 mammalian cell the opposite appears true. Studies on centrosome reorientation in migrating

215 fibroblasts suggest that the centrosome might remain relatively central while the nucleus

216 moves rearwards [20]. Centrosome position is maintained by dynein-mediated cortical

217 tethering of microtubules [62]; however, it is unclear whether centrosome rotation drives

218 nuclear movement or vice versa.

219

220 In higher eukaryotes, the link between the nuclear envelope and the centrosome is essential 221 for development [63] and is robust enough to withstand cell lysis and nuclear isolation [64].

222 This linkage is required for nuclear migration and the control of cell cycle timing [64] and in

223 Drosophila there is evidence that the centrosomes can reach the cell cortex during

224 development with the aid of the nucleus [65].

225

226 In the organisms studied to date, the centrosome-nucleus linkage is mediated by proteins containing paired KASH (Klarsicht-anchorage protein1-Syne homology) and SUN (Sad1UNC84) domains (Figure 5). First discovered in C. elegans, proteins with these domains are

229 found across eukaryotes and localise to the nuclear envelope and centrosomes [63, 66-68].

230 Multiple SUN and KASH proteins exist that provide links between the nuclear envelope and 231 cytoskeletal polymers [69-70] (Figure 5). One of the diverse roles of the SUN-KASH 232 complex is the regulation of centrosome position. The C. elegans KASH protein ZYG-12 233 anchors the centrosome to the nuclear envelope during embryogenesis [63]. ZYG-12 is not 234 found in mammalian cells; however several proteins fulfill the role of nucleus-centrosome 
235 linkers including the nuclear membrane protein emerin [71], and the multi-isoform KASH 236 protein Nesprin 2 [66]. Several Nesprin isoforms contain calponin-homology domains that

237 allow them to bind actin, and these are implicated in positioning the centrosome during 238 ciliogenesis of sensory cilia [72], suggesting that actin-dependent nuclear re-positioning or 239 rotation may re-orient the centrosome apically. A novel epithelial-specific Nesprin isoform,

240 Nesprin 4, interacts with the microtubule motor kinesin-1, and this link is proposed to 241 contribute to nucleus and centrosome positioning in interphase cells [73]. It will be 242 interesting to see if Nesprin 4 and kinesins are also involved in centrosome/centriole 243 positioning during ciliogenesis as Nesprin-microtubule links are also important in cell 244 migration. In migrating neurons, a SUN1/2-Nesprin1/2 complex acts with the lissencephaly245 associated proteins Lis 1 and Doublecortin to couple the centrosome and nucleus through cytoplasmic dynein $[66,74]$

\section{Regulation of centriole movements}

249 Given that centrioles and centrosomes can track around the nuclear envelope, change their 250 position relative to the nucleus, embed themselves in the cortex, or move around the cell, how 251 do they know where to go? In the absence of other cues, cell-cell contacts are the main 252 mediators of centrosome positioning [75-76]. The extracellular signals that trigger centrosome and centriole movements are varied; however there is now much evidence from a variety of systems that these signals converge on the Par (partitioning) proteins [77] and the Rho family of small GTPases.

257 The Par proteins are a key set of polarity proteins that were identified in screens for mutants 258 affecting the first asymmetric cell division in C. elegans [78-79]. In both C. elegans and 259 Drosophila, the Par complex acts through the Rho family of small GTPases and the actin 
cytoskeleton to establish the cortical polarity that is needed for spindle positioning $[50,80]$.

261 Once this initial polarity is established, the same mechanisms act together with microtubulecortical interactions to produce the forces that result in the asymmetrically placed spindle.

263 External signalling cues from neighbouring epithelial cells are needed to regulate the

264 localisation of polarity markers - and hence the axis of the mitotic spindle - during

265 asymmetric cell division in Drosophila neuroblasts [81]. Two Rho GTPases act together to

266 regulate polarity establishment in C. elegans. Rho1 mediates the centrosome-dependent

267 cortical actomyosin rearrangements that lead to contractile asymmetry within the cortex.

268 Cdc42 mediates the link between the cortex and Par6 proteins, and coordinates Par protein

269 segregation as the cortical asymmetry develops [80]. During cell migration, integrin

270 signalling through $\mathrm{Cdc} 42$ to Par6 and aPKC is required for the microtubule-dependent

271 centrosome localisation observed during astrocyte migration [10, 21] and blocking Cdc42

272 prevents macrophage polarization towards a chemotactic signal [82]. A Par3-Par6-aPKC

273 complex stabilises microtubule-dependent cell polarity during keratinocyte migration,

274 although its role in centrosome movement is unclear [83]. During development, aPKC is

275 needed during neuronal repolarization [84] and Pard3 controls centrosome positioning during

276 neurulation [85]. During ciliogenesis of multi-ciliated epithelia, the Par3-Par6-aPKC polarity

277 complex localises to cilia and regulates ciliogenesis via association with kinesin-II [86], one

278 of the motors required to build cilia by intraflagellar transport [43]. Rho is not needed for

279 centriole re-orientation during virological synapse formation, however, inhibition of Rac and

280 Cdc42 prevents centrioles from re-orientating [49]. Cdc42 and Par proteins are also 281 implicated in immunological synapse formation. Cdc42 inhibition blocks centrosome re282 orientation [87], while Par3 is recruited to the synapse [88] and overexpression of a 283 dominant-negative form of Par1b blocks centrosome re-orientation [89], suggesting that Par3

284 localisation is functionally relevant to immunological synapse formation. The signalling 
events that regulate the Par proteins in this case are unclear, however strength of signalling via the $\mathrm{T}$ cell receptor is important [90] and when more than one contact is present, the centrosome can oscillate between the possible targets [91] until the decision is made to kill the target that produces the strongest signal [92]. It is therefore reasonable to suggest that this provides the required external cue.

\section{Concluding remarks}

292 While the importance of the cytoskeleton, polarity proteins, and the nuclear envelope in centriole movements has long been recognised in several different fields, the idea of common

294 themes has been slower to emerge. Research carried out over the last few years has highlighted that, even though centrioles and centrosomes are positioned to achieve very different outcomes, much of the basic machinery that is used is remarkably similar. It seems likely that disparate signalling events might converge on the recruitment of the Par proteins to establish and maintain the asymmetry that is a key feature of these centrosome re-orientation events. In general, where multiple centrioles need to be moved, there is a requirement for the

300 actin cytoskeleton, while microtubule forces acting on the cell cortex are particularly important for positioning individual centrosomes, and during cell division. Finally, the involvement of KASH proteins in multiple centrosome positioning contexts suggests that they too may represent a conserved mechanism for regulating centrosome location, and their

304 potential roles in mediating other centrosome movements warrants investigation. A challenge

305 for the future is to identify the polarity cues that regulate centrosome position in organisms 306 outside the metazoa that lack the Par proteins. It will be interesting to see if there are 307 conserved mechanisms to set up asymmetry in these systems. These might include examples 308 of cytotaxis such as those that are involved in polarity replication during trypanosome 309 morphogenesis [93] or the inheritance of cortical organisation in ciliates [94]. 
311 Several proteins that are implicated in control of centrosome/centriole positioning have been

312 linked to human inherited disease. Lissencephaly, or "smooth brain," is a brain malformation

313 disorder caused by abnormal neuronal migration early in development. Two of the underlying

314 proteins, Lis 1 and Doublecortin, mediate the centrosome-nucleus linkage [74] and it seems

315 likely that polarity problems caused by disruption of this link might contribute to the disease.

316 Other neuronal migration disorders can also result in structurally abnormal or missing areas

317 of the brain including midline defects such as agenesis of the corpus callosum and hypoplasia

318 of the cerebellar vermis. Many the ciliopathies, or diseases of cilium dysfunction, present

319 with midline defects as part of the phenotype, and two ciliopathies, Meckel-Gruber syndrome

320 and hydrolethalus syndrome, have been linked to centrosome/basal body-positioning defects

321 [95-96]. How these fit in to the pathways and processes described here remains to be seen,

322 however, the Meckel-Gruber syndrome proteins are implicated in planar cell polarity

323 signaling [97-98] and may regulate centrosome re-orientation during ciliogenesis through

324 actin cytoskeleton remodeling and maintaining the centrosome-nuclear envelope connection

325 [72]. Finally, there are other diseases that have been linked to centrosome dysfunction [99]

326 and it will be fascinating to discover if centriole/centrosome position is also compromised in

327 these cases.

328

329 As more details of the molecular control of polarity establishment are uncovered, it will

330 become possible to understand which of the activities in C. elegans represent general 331 principles in polarity establishment, and which are specialized to the particular case of 332 embryonic polarity establishment. Despite the likelihood of cell-type specific specializations, 333 analysis reveals a commonality in the mechanisms used to move centrioles and centrosomes

334 throughout eukaryotic biology. Notwithstanding the very different contexts in which centriole 
movements are observed, these commonalities have the potential to contribute to our

understanding of centriole movements in less well-studied systems.

\section{References}

3391 Rios, R.M. and Bornens, M. (2003) The Golgi apparatus at the cell centre. Curr Opin Cell 340 Biol 15, 60-66

3412 Griffiths, G.M., et al. (2010) The immunological synapse: a focal point for endocytosis and 342 exocytosis. J Cell Biol 189, 397-406

3433 Prigozhina, N.L. and Waterman-Storer, C.M. (2004) Protein kinase D-mediated anterograde membrane trafficking is required for fibroblast motility. Curr Biol 14, 88-98 4 Karsenti, E. and Nedelec, F. (2004) The mitotic spindle and actin tails. Biol Cell 96, 237240

5 Mahoney, N.M., et al. (2006) Making microtubules and mitotic spindles in cells without functional centrosomes. Curr Biol 16, 564-569

6 Walczak, C.E., et al. (1998) A model for the proposed roles of different microtubule-based motor proteins in establishing spindle bipolarity. Curr Biol 8, 903-913

7 Cowan, C.R. and Hyman, A.A. (2004) Centrosomes direct cell polarity independently of microtubule assembly in C. elegans embryos. Nature 431, 92-96

8 Vaughan, S. and Gull, K. (2003) The trypanosome flagellum. J Cell Sci 116, 757-759

9 Feldman, J.L., et al. (2007) The mother centriole plays an instructive role in defining cell geometry. PLoS Biol 5, e149

10 Etienne-Manneville, S. and Hall, A. (2001) Integrin-mediated activation of Cdc42 controls cell polarity in migrating astrocytes through PKCzeta. Cell 106, 489-498

11 Manneville, J.B. and Etienne-Manneville, S. (2006) Positioning centrosomes and spindle poles: looking at the periphery to find the centre. Biol Cell 98, 557-565

36012 Palazzo, A.F., et al. (2001) Cdc42, dynein, and dynactin regulate MTOC reorientation independent of Rho-regulated microtubule stabilization. Curr Biol 11, 1536-1541

363

364

365

366

367 13 Tsai, J.W., et al. (2007) Dual subcellular roles for LIS1 and dynein in radial neuronal migration in live brain tissue. Nat. Neurosci. 10, 970-979

14 Stinchcombe, J.C., et al. (2006) Centrosome polarization delivers secretory granules to the immunological synapse. Nature 443, 462-465

15 Piguet, V. and Sattentau, Q. (2004) Dangerous liaisons at the virological synapse. J Clin Invest 114, 605-610

16 Basto, R., et al. (2006) Flies without centrioles. Cell 125, 1375-1386

17 Dawe, H.R., et al. (2007) Centriole/basal body morphogenesis and migration during ciliogenesis in animal cells. J Cell Sci 120, 7-15

37118 Euteneuer, U. and Schliwa, M. (1984) Persistent, directional motility of cells and

372 cytoplasmic fragments in the absence of microtubules. Nature 310, 58-61

37319 Koonce, M.P., et al. (1999) Dynein motor regulation stabilizes interphase microtubule arrays and determines centrosome position. Embo J 18, 6786-6792

20 Gomes, E.R., et al. (2005) Nuclear movement regulated by Cdc42, MRCK, myosin, and actin flow establishes MTOC polarization in migrating cells. Cell 121, 451-463

21 Etienne-Manneville, S. and Hall, A. (2003) Cdc42 regulates GSK-3beta and adenomatous polyposis coli to control cell polarity. Nature 421, 753-756 22 Burakov, A., et al. (2003) Centrosome positioning in interphase cells. J Cell Biol 162, 963-969 
23 Gonczy, P., et al. (1999) Cytoplasmic dynein is required for distinct aspects of MTOC positioning, including centrosome separation, in the one cell stage Caenorhabditis elegans embryo. J Cell Biol 147, 135-150

24 Robinson, J.T., et al. (1999) Cytoplasmic dynein is required for the nuclear attachment and migration of centrosomes during mitosis in Drosophila. J Cell Biol 146, 597-608

25 Piel, M., et al. (2000) The respective contributions of the mother and daughter centrioles to centrosome activity and behavior in vertebrate cells. J Cell Biol 149, 317-329

26 Chevrier, V., et al. (2002) The Rho-associated protein kinase p160ROCK is required for centrosome positioning. J Cell Biol 157, 807-817

27 Riento, K. and Ridley, A.J. (2003) Rocks: Multifunctional kinases in cell behaviour. Nat Rev Mol Cell Biol 4, 446-456

28 Magdalena, J., et al. (2003) Microtubule involvement in NIH 3T3 Golgi and MTOC polarity establishment. J Cell Sci 116, 743-756

29 Sonneville, R. and Gonczy, P. (2004) Zyg-11 and cul-2 regulate progression through meiosis II and polarity establishment in C. elegans. Development 131, 3527-3543

30 Tsai, M.C. and Ahringer, J. (2007) Microtubules are involved in anterior-posterior axis formation in C. elegans embryos. J Cell Biol 179, 397-402

31 Boisvieux-Ulrich, E., et al. (1989) In vitro effects of colchicine and nocodazole on ciliogenesis in quail oviduct. Biol Cell 67, 67-79

32 Klotz, C., et al. (1986) Myosin at the apical pole of ciliated epithelial cells as revealed by a monoclonal antibody. J Cell Biol 103, 613-619

33 Lemullois, M., et al. (1988) Development and functions of the cytoskeleton during ciliogenesis in metazoa. Biol Cell 63, 195-208

34 Lemullois, M., et al. (1987) Immunocytochemical localization of myosin during ciliogenesis of quail oviduct. Eur J Cell Biol 43, 429-437

35 Boisvieux-Ulrich, E., et al. (1990) Cytochalasin D inhibits basal body migration and ciliary elongation in quail oviduct epithelium. Cell Tissue Res 259, 443-454

36 Boisvieux-Ulrich, E., et al. (1987) In vitro effects of benzodiazepines on ciliogenesis in the quail oviduct. Cell Motil Cytoskeleton 8, 333-344

37 Gray, R.S., et al. (2009) The planar cell polarity effector Fuz is essential for targeted membrane trafficking, ciliogenesis and mouse embryonic development. Nat Cell Biol 11, $1225-1232$

38 Park, T.J., et al. (2006) Ciliogenesis defects in embryos lacking inturned or fuzzy function are associated with failure of planar cell polarity and Hedgehog signaling. Nat Genet 38, 303311

39 Park, T.J., et al. (2008) Dishevelled controls apical docking and planar polarization of basal bodies in ciliated epithelial cells. Nat Genet 40, 871-879

40 Pan, J., et al. (2007) RhoA-mediated apical actin enrichment is required for ciliogenesis and promoted by Foxj1. J Cell Sci 120, 1868-1876

41 Hirota, Y., et al. (2010) Planar polarity of multiciliated ependymal cells involves the anterior migration of basal bodies regulated by non-muscle myosin II. Development

42 Griffiths, G.M., et al. (2010) The immunological synapse: a focal point for endocytosis and exocytosis. J Cell Biol 189, 399-406

43 Scholey, J.M. (2003) Intraflagellar transport. Ann Rev Cell Dev Biol 19, 423-443

44 Finetti, F., et al. (2009) Intraflagellar transport is required for polarized recycling of the TCR/CD3 complex to the immune synapse. Nat Cell Biol 11, 1332-1339

45 Ryser, J.E., et al. (1982) The area of attachment of cytotoxic lymphocytes to their target cells shows high motility and polarisation of actin, but not myosin. J Immunol 128, 11591162 
46 Stinchcombe, J.C., et al. (2001) The immunological synapse of CTL contains a secretory domain and membrane bridges. Immunity 15, 751-761

47 Jolly, C., et al. (2004) HIV-1 cell to cell transfer across an env-induced, actin-dependent synapse. J Exp Med 199, 283-293

48 Jolly, C., et al. (2007) Requirement for an intact T-cell actin and tubulin cytoskeleton for efficient assembly and spread of human immunodeficiency virus type 1 . J Virol 81, 55475560

49 Nejmeddine, M., et al. (2005) Human T-lymphotropic virus, type 1, tax protein triggers microtubule reorientation in the virological synapse. J Biol Chem 280, 29653-29660

50 Kunda, P. and Baum, B. (2009) The actin cytoskeleton in spindle assembly and positioning. Trends Cell Biol 19, 174-179

51 Yamashita, Y.M., et al. (2007) Asymmetric inheritance of mother versus daughter centrosome in stem cell division. Science 315, 518-521

52 Carreno, S., et al. (2008) Moesin and its activating kinase Slik are required for cortical stability and microtubule organization in mitotic cells. J Cell Biol 180, 739-746

53 Kunda, P., et al. (2008) Moesin controls cortical rigidity, cell rounding, and spindle morphogenesis during mitosis. Curr Biol 18, 91-101

54 Rosenblatt, J., et al. (2004) Myosin II-dependent cortical movement is required for centrosome separation and positioning during mitotic spindle assembly. Cell 117, 361-372

55 Thery, M., et al. (2007) Experimental and theoretical study of mitotic spindle orientation. Nature 447, 493-U496

56 Toyoshima, F. and Nishida, E. (2007) Integrin-mediated adhesion orients the spindle parallel to the substratum in an EB1-and myosin X-dependent manner. Embo J 26, 14871498

57 Woolner, S., et al. (2008) Myosin-10 and actin filaments are essential for mitotic spindle function. J Cell Biol 182, 77-88

58 Gull, K. (1999) The cytoskeleton of trypanosomatid parasites. Annu Rev Microbiol 53, $629-655$

59 Robinson, D.R. and Gull, K. (1991) Basal body movements as a mechanism for mitochondrial genome segregation in the trypanosome cell cycle. Nature 352, 731-733

60 Brugerolle, G. and Mignot, J.P. (2003) The rhizoplast of chrysomonads, a basal bodynucleus connector that polarises the dividing spindle. Protoplasma 222, 13-21

61 Wright, R.L., et al. (1985) A nucleus-basal body connector in Chlamydomonas reinhardtii that may function in basal body localization or segregation. J Cell Biol 101, 1903-1912

62 Schmoranzer, J., et al. (2009) Par3 and dynein associate to regulate local microtubule dynamics and centrosome orientation during migration. Curr Biol 19, 1065-1074

63 Malone, C.J., et al. (2003) The C. elegans hook protein, ZYG-12, mediates the essential attachment between the centrosome and nucleus. Cell 115, 825-836

64 Bornens, M. (2008) Organelle positioning and cell polarity. Nat Rev Mol Cell Biol 9, 874886

65 Raff, J.W. and Glover, D.M. (1989) Centrosomes, and not nuclei, initiate pole cell formation in Drosophila embryos. Cell 57, 611-619

66 Zhang, X., et al. (2009) SUN1/2 and Syne/Nesprin-1/2 complexes connect centrosome to the nucleus during neurogenesis and neuronal migration in mice. Neuron 64, 173-187

67 Zhen, Y.Y., et al. (2002) NUANCE, a giant protein connecting the nucleus and actin cytoskeleton. J Cell Sci 115, 3207-3222

68 Zhang, Q., et al. (2001) Nesprins: a novel family of spectrin-repeat-containing proteins that localize to the nuclear membrane in multiple tissues. J Cell Sci 114, 4485-4498

69 Starr, D.A. and Fischer, J.A. (2005) KASH 'n Karry: the KASH domain family of cargospecific cytoskeletal adaptor proteins. Bioessays 27, 1136-1146 
70 Worman, H.J. and Gundersen, G.G. (2006) Here come the SUNs: a nucleocytoskeletal missing link. Trends Cell Biol 16, 67-69

71 Salpingidou, G., et al. (2007) A novel role for the nuclear membrane protein emerin in association of the centrosome to the outer nuclear membrane. J Cell Biol 178, 897-904

72 Dawe, H.R., et al. (2009) Nesprin-2 interacts with meckelin and mediates ciliogenesis via remodelling of the actin cytoskeleton. J Cell Sci 122, 2716-2726

73 Roux, K.J., et al. (2009) Nesprin 4 is an outer nuclear membrane protein that can induce kinesin-mediated cell polarization. Proc Nat Acad Sci U.S.A. 106, 2194-2199

74 Tanaka, T., et al. (2004) Lis1 and doublecortin function with dynein to mediate coupling of the nucleus to the centrosome in neuronal migration. J Cell Biol 165, 709-721

75 Dupin, I., et al. (2009) Classical cadherins control nucleus and centrosome position and cell polarity. J Cell Biol 185, 779-786

76 Desai, R.A., et al. (2009) Cell polarity triggered by cell-cell adhesion via E-cadherin. J. Cell Sci 122, 905-911

77 Goldstein, B. and Macara, I.G. (2007) The PAR proteins: fundamental players in animal cell polarization. Dev Cell. Nov;13(5):609-22.

78 Kemphues, K.J., et al. (1988) Identification of genes required for cytoplasmic localization in early C. elegans embryos. Cell 52, 311-320

79 Tabuse, Y., et al. (1998) Atypical protein kinase C cooperates with PAR-3 to establish embryonic polarity in Caenorhabditis elegans. Development 125, 3607-3614

80 Cowan, C.R. and Hyman, A.A. (2007) Acto-myosin reorganization and PAR polarity in C-elegans. Development 134, 1035-1043

81 Siegrist, S.E. and Doe, C.Q. (2006) Extrinsic cues orient the cell division axis in Drosophila embryonic neuroblasts. Development 133, 529-536

82 Allen, W.E., et al. (1998) A role for Cdc42 in macrophage chemotaxis. J. Cell Biol. 141, 1147-1157

83 Pegtel, D.M., et al. (2007) The Par-Tiam1 complex controls persistent migration by stabilizing microtubule-dependent front-rear polarity. Curr Biol 17, 1623-1634

84 Higginbotham, H., et al. (2006) GSK3 beta and PKC zeta function in centrosome localization and process stabilization during Slit-mediated neuronal repolarization. Mol. Cell. Neurosci. 32, 118-132

85 Hong, E., et al. (2010) The Polarity Protein Pard3 is Required for Centrosome Positioning During Neurulation. Development

86 Fan, S.L., et al. (2004) Polarity proteins control ciliogenesis via kinesin motor interactions. Curr. Biol. 14, 1451-1461

87 Stowers, L., et al. (1995) Regulation of the polarization of T cells toward antigenpresenting cells by Ras-related GTPase CDC42. Proc. Nat. Acad. Sci. U.S.A. 92, 5027-5031

88 Ludford-Menting, M.J., et al. (2005) A network of PDZ-containing proteins regulates T cell polarity and morphology during migration and immunological synapse formation. Immunity 22, 737-748

89 Lin, J., et al. (2009) The Polarity Protein Par1b/EMK/MARK2 Regulates T Cell ReceptorInduced Microtubule-Organizing Center Polarization. J. Immunol. 183, 1215-1221

90 Jenkins, M.R., et al. (2009) The Strength of T Cell Receptor Signal Controls the Polarization of Cytotoxic Machinery to the Immunological Synapse. Immunity 31, 621-631

91 Kuhn, J.R. and Poenie, M. (2002) Dynamic polarization of the microtubule cytoskeleton during CTL-Mediated killing. Immunity 16, 111-121

92 Wiedemann, A., et al. (2006) Cytotoxic T lymphocytes kill multiple targets simultaneously via spatiotemporal uncoupling of lytic and stimulatory synapses. Proc. Nat. Acad. Sci. U.S.A. 103, 10985-10990 
93 Moreira-Leite, F.F., et al. (2001) A trypanosome structure involved in transmitting cytoplasmic information during cell division. Science 294, 610-612

94 Beisson, J. and Sonneborn, T.M. (1965) Cytoplasmic Inheritance of the Organization of the Cell Cortex in Paramecium Aurelia. Proc. Nat. Acad. Sci U.S.A. 53, 275-282

95 Dammermann, A., et al. (2009) The hydrolethalus syndrome protein HYLS-1 links core centriole structure to cilia formation. Genes Dev 23, 2046-2059

96 Dawe, H.R., et al. (2007) The Meckel-Gruber Syndrome proteins MKS1 and meckelin interact and are required for primary cilium formation. Hum. Mol. Genet. 16, 173-186

97 Kim, S.K., et al. (2010) Planar Cell Polarity Acts Through Septins to Control Collective Cell Movement and Ciliogenesis. Science

98 Valente, E.M., et al. (2010) Mutations in TMEM216 perturb ciliogenesis and cause Joubert, Meckel and related syndromes. Nat. Genet. 42, 619-625

99 Nigg, E.A. and Raff, J.W. (2009) Centrioles, centrosomes, and cilia in health and disease. Cell 139, 663-678

100 Tsou, M.F. and Stearns, T. (2006) Mechanism limiting centrosome duplication to once per cell cycle. Nature 442, 947-951

\section{Figure Legends}

Fig 1: Centriole duplication cycle. During interphase/G1 centrioles function in organizing microtubules, and in many eukaryotic cells the mature centriole assembles a primary cilium/flagellum. The centriole pair must duplicate only once during the cell cycle and this begins at the G1/S-phase transition with a pro-centriole assembled orthogonal to each mature centriole. Recent studies have dissected the molecules required for initial pro-centriole assembly in C. elegans early embryos and humans (pro-centriole assembly: C. elegans: SPD2, ZYG-1 Sas-6, Sas-5, Sas-4, $\alpha-, \beta-$, and $\gamma$-tubulin; Humans: Cep192, Plk4/Sak, Sas-6, Sas4, CPAP, $\alpha-, \beta-, \gamma-, \delta$-, $\varepsilon$-tubulin, CP110, and Cep135. Regulatory molecules: Cdk1/Cyclin B, Aurora-A, Plks. Control of centriole duplication: Separase, Plk1, Plk4, SAS-6.) [99]. Procentriole elongation continues through G2 until there are two pairs of centrioles that migrate to the pole of the spindle. Following mitosis the tight association and orthogonal orientation of the mature centriole and pro-centriole is no longer apparent. This is a stage termed 'disengagement' and is a crucial stage in the control of centriole duplication [100]. (a) Microtubule arrays/primary cilium functions. (b) New centriole formation. (c) New centriole 
elongation. (d) Centrosome segregation. (e) Centrosomes move to spindle poles. (f)

563 Centrosome inheritance to daughter cells.

564

565 Fig 2: Centrosome migration in G1/interphase cells. In many animal cells the centrosome

566 migrates from a central position within the cell to the cell cortex, where a primary cilium

567 assembles from the mature centriole. The primary cilium acts as an antenna for the cell that senses the environment and is needed to transduce certain signaling pathways. Migration of the centrosome can involve interaction with radial microtubules and actomyosin at the cell cortex, however, the role of the cortex in primary cilium formation is not known. (a) G1/interphase cell. The centrosome is located centrally within the cell. Central location is maintained by microtubules/dynein and regulated by p160Rock. (b) The centrosome moves to the cell surface in some cell types via interaction of microtubules with the actomyosin cell cortex. (c) A microtubule-based primary cilium is assembled from the mature centriole.

Fig 3: Centrosome/basal body migration during cell division. (a). Symmetric \& asymmetric cell division. Migration of the duplicated centrosomes to the opposite poles of the spindle requires actin-microtubule interactions with the cell cortex (left). The Par proteins are important in modulating these interactions in order to promote asymmetric positioning (right). (b). Interphase African trypanosome cell with a single flagellum assembled from the mature basal body (left). G1/S-phase basal body duplication occurs and a new flagellum assembles alongside the old flagellum. Migration is microtubule-dependent via subpellicular microtubules at the cell cortex (middle). Intriguingly, actin and myosin II are not involved in either basal body migration or cytokinesis in T. brucei (right). (c). Bi-flagellated interphase C. reinhardtii cell (left). Flagella are cleaved and the centrioles migrate to the poles of the mitotic spindle via a nucleus-centriole connector (rhizoplast; middle). Centrioles return to 
the cell cortex and two new flagella are assembled for each daughter cell prior to cytokinesis

588 (right).

589

$590 \quad$ Fig. 4: Centriole/basal body migration in terminally differentiated cells. (a). The process

591 of ciliogenesis produces thousands of motile or immotile cilia on many specialized terminally

592 differentiated cells. Large numbers of basal bodies are formed within a single cell (left).

593 Basal bodies migrate and dock with the cell membrane. Movement requires actomyosin, and

594 is regulated by GTPase RhoA (middle). Motile or immotile cilia are assembled from the 595 docked basal bodies (right). Basal bodies form via a combination of the centriolar and 596 acentriolar pathways (see text box). The role of the existing centrosome is unknown (b).

597 Cytotoxic T-cells form an immunological synapse to facilitate killing a target cell.

598 Centrosome migration is required during the early stages of synapse formation and occurs by

599 interactions between microtubules and the cell cortex. Recognition of a target cell by a

600 cytotoxic T-cell and assembly of the synapse (left; arrow points to nascent synapse).

601 Movement of the centrosome to the synapse requires both microtubules and actomyosin

602 (middle). The centrosome docks at the plasma membrane of the immunological synapse and lytic granules (black) travel along microtubules to the synapse to kill the target cell (right).

604

605

606

Fig 5: SUN and KASH domain proteins couple the nucleus to the actin and microtubule

607 the inner and outer nuclear membranes. Many different KASH-domain proteins exist and can cytoskeletons. The SUN domain-KASH domain interaction occurs within the space between 608 provide a physical linkage between the nuclear lamina and the cytoskeleton. KASH proteins 609 with an N-terminal actin-binding domain link the actin cytoskeleton to the nucleus. Other 610 KASH proteins link microtubules to the nucleus via interactions with kinesin or dynein. The 
611 SUN-KASH interaction is evolutionarily conserved and ha many roles within cells,

612 including nuclear migration and centrosome orientation.

613

$614 \quad$ Figure I

615 Transmission electron micrograph of a centriole pair from a mouse kidney cell. Scale bar:

$616100 \mathrm{~nm}$.

617

618 Glossary:

619 Actin motors: myosins are actin motors that carry cargo along actin and are ATP-dependent.

620 With the exception of myosin VI all other myosins studied to date are plus-end directed.

621 Astral microtubules: extend out from each centrosome at opposite poles of the mitotic 622 spindle pole to the cell cortex and are required for mitotic spindle orientation.

623 Basal body: a microtubule organizing centre that subtends a cilium or flagellum.

624 Cell cortex: a specialized area of the cell underlying the plasma membrane that is required 625 for mechanical support of cell shape and form. Microtubules (called cortical microtubules), 626 actin (called cortical actin) or both are found at the cell cortex in a wide range of eukaryotic 627 cells.

628 Centriole: a microtubule-based barrel-shaped structure generally composed of 9 triplet 629 microtubules that is found in many cells (Figure I).

630 Centrosome: the major microtubule organizing centre in mammalian cells. It organizes 631 radial arrays of microtubules, mitotic spindle microtubules and astral microtubules, and 632 contains a pair of centrioles.

633 Immunological/Virological synapse: named for their similarity to classical neurological 634 synapses, the immunological synapse is the interface between an antigen-presenting cell and 
635 a lymphocyte, while the virological synapse is the interface between infected cells and target 636 cells that can mediate cell-cell spread of viruses.

637 Microtubule motors: kinesin motors move along microtubules towards the plus-end of 638 microtubules and dynein motors move towards the minus-end of microtubules. Both are 639 ATP-dependent motors.

640 Pericentriolar material: the matrix that surrounds the centrioles within the centrosome. It 641 contains proteins responsible for microtubule nucleation and anchoring and plays a role in 642 centrosome duplication.

\section{Box 1. Basal body production during ciliogenesis}

645 Many metazoan organisms build two types of cilium: non-motile sensory, or primary, cilia 646 and motile cilia. Each assembles from a basal body, which is analogous to the mitotic 647 centrioles.

648

649 - Primary cilia are solitary organelles that assemble from a basal body derived from the 650 pre-existing mature centriole, which moves to the cell surface and docks before extending 651 the ciliary axoneme.

652

653 In contrast, there can be hundreds of motile cilia on a single cell and each needs a basal body.

654 Basal body formation is linked to differentiation rather than proliferation and multiple basal 655 bodies are formed in the cytoplasm and then simultaneously migrate to the cell surface. Basal 656 bodies are formed de novo by a combination of the centriolar pathway and the acentriolar 657 pathway, both of which can occur in a single cell. 
- In the centriolar pathway, new basal bodies are produced around an existing centriole 659 template, just as observed during cell cycle-dependent centriole duplication. However, 660 more than one new basal body can form around a single centriole.

661 - In the acentriolar pathway, basal body formation is not templated. Here, multiple basal 662 bodies form around an intermediary structure called a deuterosome rather than around an 663 existing centriole.

664 
(a)

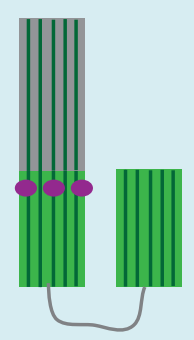

G1/interphase (b)

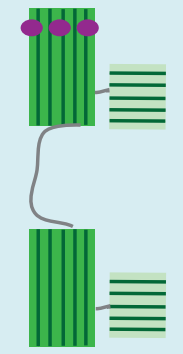

(c)
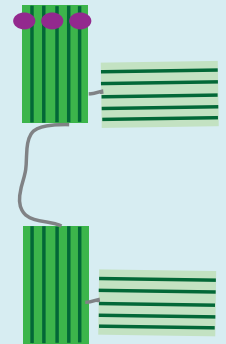

(d)

(e)

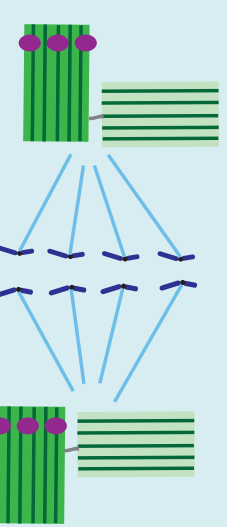

M-phase (f)
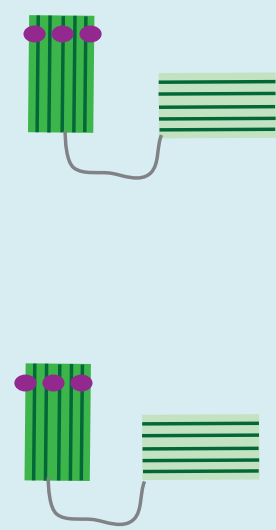

late M-phase/G1

Key:

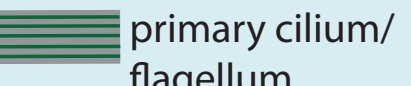
flagellum

Figure 1 


\section{Centrosome migration during G1/interphase}

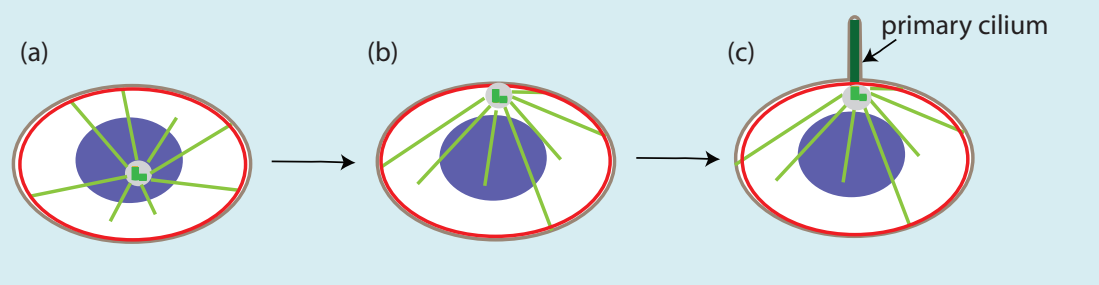

Key: centrosome

plasma nucleus membrane

flagellum/cilium radial microtubule actomyosin

Figure 2 


\section{Centrosome/basal body migration during cell division}

(a) symmetric \& asymmetric cell division
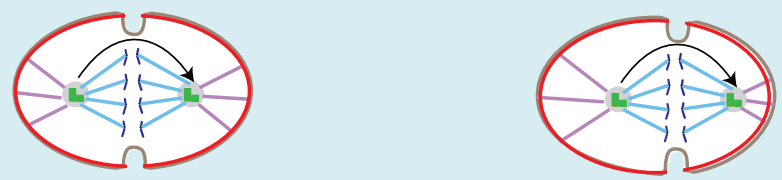

(b) basal body migration in Trypanosoma brucei
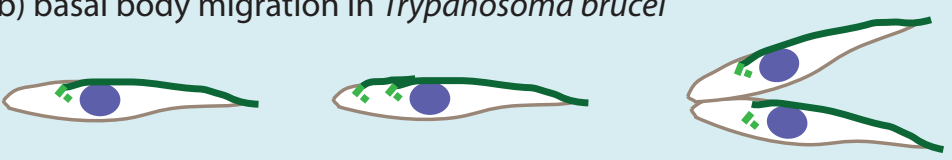

(c) centriole migration in Chlamydomonas reinhardtii
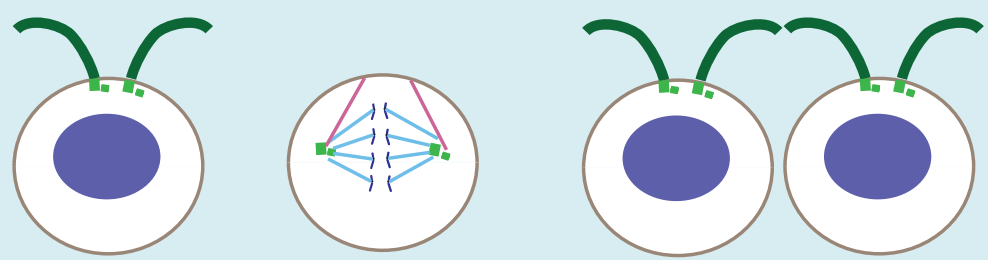

Key: centrosome plasma

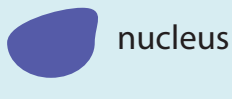

membrane

flagellum/cilium actomyosin spindle microtubule

S sister chromatid nucleus-centriole connection (rhizoplast)

Figure 3 


\section{Centriole/basal body migration in terminally differentiated cells}

(a) basal body migration during ciliogenesis
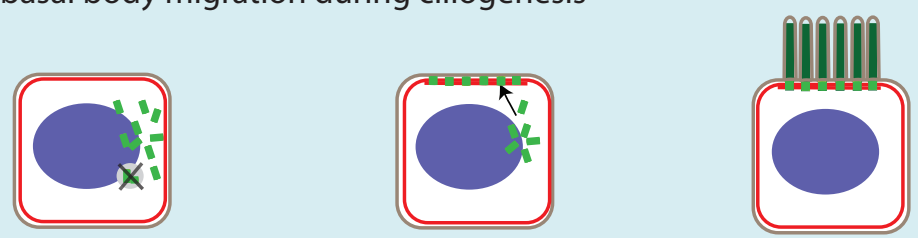

(b) immunological synapse formation
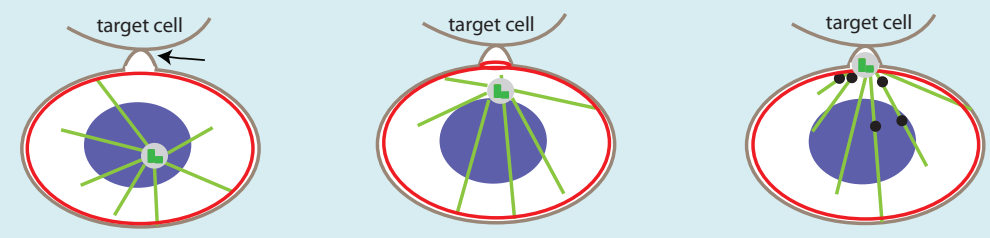

Key: centrosome radial microtubule actomyosin

flagellum/cilium nucleus basal body

Figure 4 


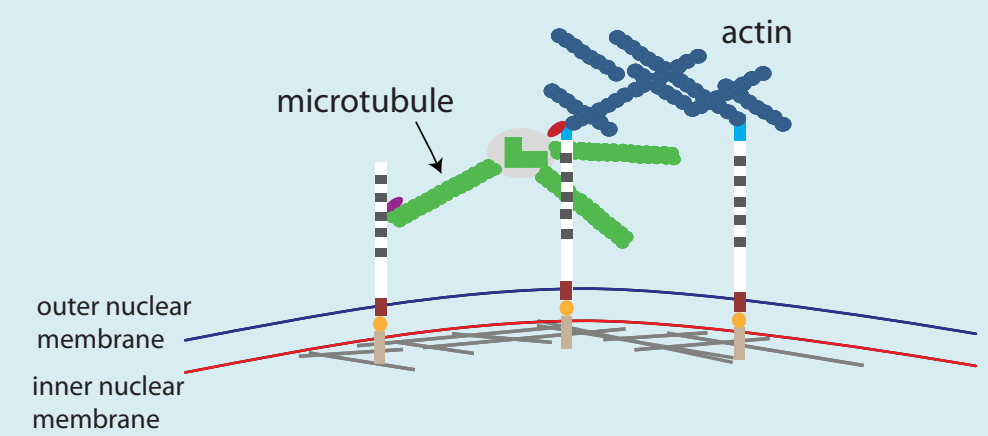

Key: centrosome dynein/dynactin kinesin - KASH domain protein - KASH domain — nuclear lamina

- actin-binding domain $\quad$ SUN domain protein $\quad$ SUN domain

Figure 5 


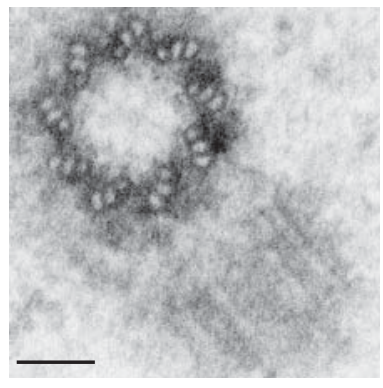

